\title{
Methods for Construction and Research Stereometric Visual Images of Multi-criteria Alternatives*
}

\author{
Alena Zakharova ${ }^{1,2[0000-0003-4221-7710]}$ and Dmitriy Korostelyov ${ }^{1,2[0000-0002-0853-7940]}$ \\ ${ }^{1}$ Keldysh Institute of Applied Mathematics Russian Academy of Sciences, Moscow, Russia \\ ${ }^{2}$ Bryansk State Technical University, Bryansk, Russia \\ zaa@tu-bryansk.ru, nigm85@mail.ru
}

\begin{abstract}
The article discusses methods for constructing and research stereometric visual images of multi-criteria alternatives. The difficulty of applying traditional decision-making methods when choosing the best alternative in the case of decision-making tasks with a large dimension is noted. Methods are proposed for reducing the dimension of a decision-making problem by constructing stereometric visual images that describe choice set and criteria set. The tasks of visual analytics are highlighted in the course of reducing the dimension of the decisionmaking task: grouping and ranking criteria, pairwise comparison and filtering of alternatives. A description of the methods for constructing stereometric visual images of multi-criteria alternatives based on histograms of alternatives and criteria is given. Also describes a method for constructing surface of the criteria's correlation. Using the developed method of alternatives visualization as a single image enables their visual filtering. The application method of the criteria visualization as a single image in combination with the construction and analysis of surface of the criteria's correlation allows for the grouping and ranking of criteria. The experiment is described on the application of the proposed methods for constructing and studying stereometric visual images of multi-criteria alternatives using the example of the problem of choosing the best OpenFoam solver, with the help of which estimates of the accuracy of calculating the inviscid flow around the cone were obtained. It is shown how, due to the above visualization methods, it is possible to reduce the count of criteria by several times.
\end{abstract}

Keywords: Alternatives Visualization, Multi-criteria Alternatives, Decisionmaking Tasks Dimension Decrease, Criteria's Convolution, Alternatives Visual Filtering.

\section{Introduction}

In various subject areas, managers and researchers are often faced with the task of choosing the best option from the many possible alternatives. There may be only a few

Copyright (C) 2020 for this paper by its authors. Use permitted under Creative Commons License Attribution 4.0 International (CC BY 4.0).

* The work was supported by Russian Science Foundation grant № 18-11-00215 
such options, or there may be hundreds (or thousands). Moreover, each option, in turn, can also be characterized by both just a few criteria, and dozens or even hundreds. Usually, this choice problem is considered as a decision-making problem [1]. When the alternatives and criteria a bit, then the decision-maker (DM) can successfully use the well-known methods of decision making: SMART, PROMETHEE, AHP, etc. [2].

In the case when we are faced with a large count of alternatives or criteria that characterize them, the use of traditional methods already becomes difficult. In such a situation, one usually resorts to methods of filtering alternatives and the method of decreasing a dimension of them. However, this is not always effective, since decision-making is a subjective and creative process, and if the above methods are applied, we can lose some important alternatives and criteria, without even noticing it. Therefore, it is important to also involve decision-makers at the stage of compressing the available set of alternatives and criteria.

The decision-maker is often unable to perceive large volumes of multi-criteria alternatives without using special tools. The decision-maker can effectively and, which is also important, holistically perceive large volumes of information only with the use of specialized methods of data visualization [3-6].

If the decision-maker is involved in the procedure of filtering alternatives and reducing their count and dimension, he faces the following tasks: criteria's grouping, criteria's ranking, pairwise comparison of alternatives $[7,8]$. Effective 3D visualization methods can be used to solve these problems. The development and improvement of such methods will allow us to study a choice set and set of criteria in order to select the most informative ones for achieving visualization goals. Consider several methods to construct and research such stereometric visual images of alternatives to filter and reduce the dimension.

\section{Methods for Constructing and Researching Stereometric Images of Multi-criteria Alternatives}

To increase the information content of stereometric images of multi-criteria alternatives, it is proposed to solve the following tasks:

1. Ranking of criteria's.

2. Grouping of criteria's.

3. Filtering of alternatives.

Suppose we have the choice set $A=\left\{A_{l}, A_{2}, \ldots, A_{N}\right\}$, where $N$ - count of alternatives. Each alternative is specified by a vector of normalized criteria values: $A_{i}=\left\{a_{i, j}\right\}$, where $1 \leq j \leq K, K$ - count of criteria, $0 \leq a_{i, j} \leq 1$. Under criterion commonly, understood parameter (quantitative or qualitative) that characterized the alternative (can be obtained by calculation, empirically, or synthetically). In the case of using qualitative criteria, an additional procedure for their normalization using specialized scales is required [8]. At the same time, higher values of the criteria $a_{i, j}$ corresponds to a more priority alternative. 
Consider several methods for visualizing multi-criteria alternatives that can be used in the course of visual analytics in solving the above problems.

\subsection{Methods for Visualizing Multi-criteria Alternatives}

There are various methods for 2D imaging of single multi-criteria alternative [9]: Histograms, 2D plots, radar plots, radial plots, etc. The simplest method for visualizing many alternatives in three-dimensional space is to place two-dimensional images of alternatives along the 3rd coordinate axis (Fig. 1).

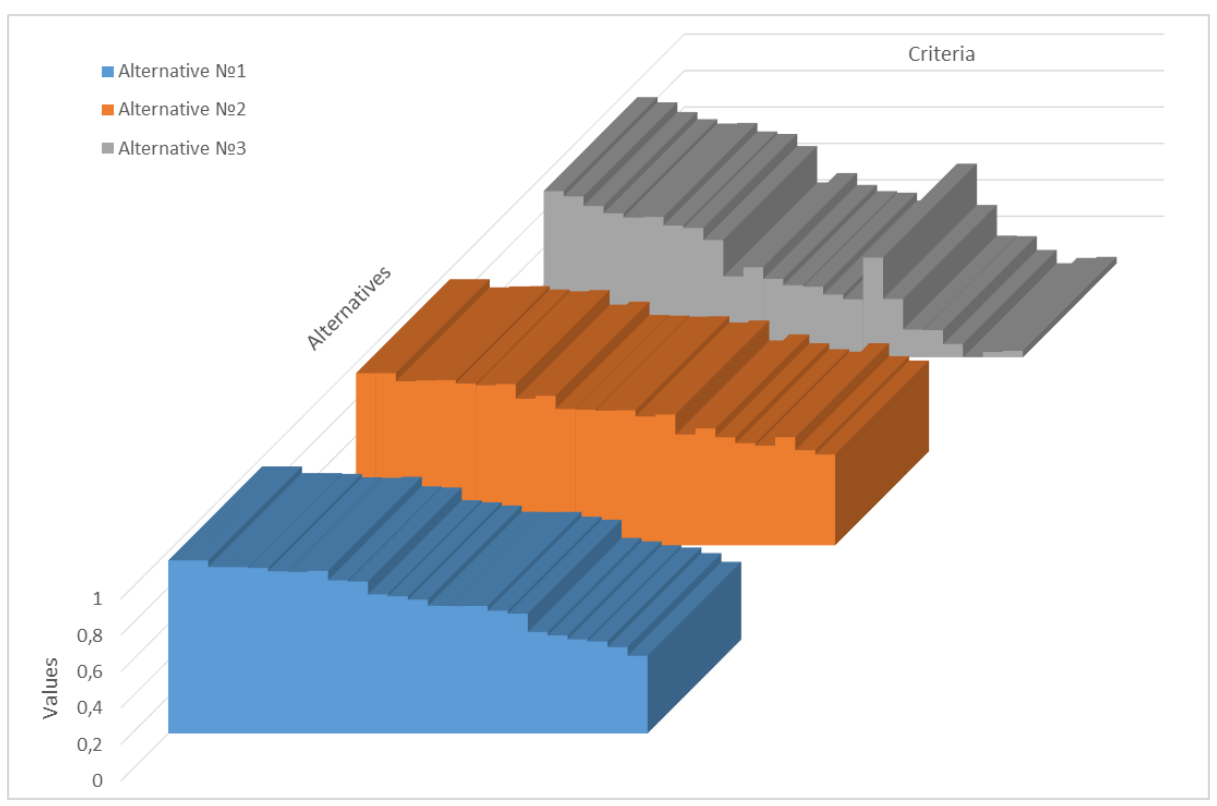

Fig. 1. Stereometric visualization of three multi-criteria alternatives using histograms

The use of this method can be effective in the case when the count of alternatives is small and the count of criteria is large, and the task is to filter out alternatives. Based on the obtained stereometric image, it is possible to visually determine the "strong" and "weak" alternatives due to the prevalence of columns with large values. However, in the case of similar characteristics of the initial data, it would be more appropriate to try to reduce the count of criteria. This would allow the use of traditional decision-making methods. However, this visualization method will not allow you to do this, because it is not obvious how the count of criteria can be reduced. Therefore, we will consider other methods of visualizing stereometric images of multi-criteria alternatives.

One possible method is to swap the criteria and alternatives for the previous visualization method - i.e. each row visualizes the values of a single criterion for all alternatives (Fig. 2). In this case, a reduction in the number of criteria can be achieved by excluding criteria that have a similar histogram with other criteria. However, the effectiveness of 


\section{A. Zakharova, D. Korostelyov}

this procedure depends on the relative position of the criteria along the axis of the criteria (Fig. 2).

Another visualization method for searching similar criteria at the given choice set can be basis on calculating correlation coefficients of the criteria values. To do this, you must first calculate the matrix of correlation coefficients between all pairs of criteria: $M=\left\{m_{i, j}\right\}$, where $1 \leq i, j \leq K, m_{i, j}$ - correlation coefficient between the criteria's values of the alternative. As a coefficient in the simplest case, you can use the Pearson correlation coefficient, calculated by the formula [10]:

$$
\begin{gathered}
m_{i, j}=\frac{\sum_{t=1}^{N}\left(a_{t, i}-\overline{a_{i}}\right) \cdot\left(a_{t, j}-\overline{a_{j}}\right)}{\sqrt{\sum_{t=1}^{N}\left(a_{t, i}-\overline{a_{i}}\right)^{2}} \cdot \sum_{t=1}^{N}\left(a_{t, j}-\overline{a_{j}}\right)^{2}}, \text { where } \\
\overline{a_{i}}=\frac{1}{N} \sum_{t=1}^{N} a_{t, i} .
\end{gathered}
$$

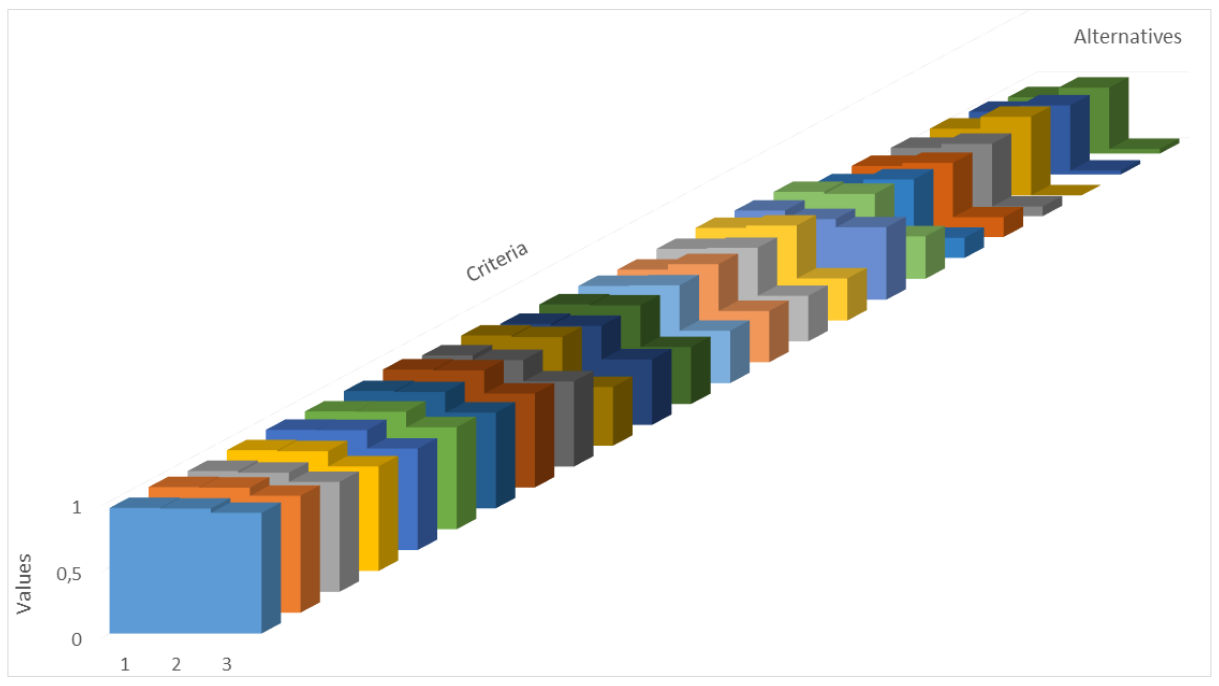

Fig. 2. Stereometric visualization of criteria for three alternatives using histograms

The thus obtained matrix $\mathrm{M}$ can be visualized as a surface (Fig. 3). A larger value of the correlation coefficient means that the criteria are very similar and one of them can be removed from consideration. As seen in Fig. 3, areas with a high cross-correlation coefficient may form on the surface, containing groups of criteria. The detection of such zones may indicate the advisability of replacing the group of criteria that form it with a single criterion.

It is worth noting that the given method (Fig. 3) can also be applied in cases where the count of alternatives and criteria is large. However, it can only be used to group and rank criteria, but it does not provide filtering of alternatives. It is also worth noting that an additional advantage of this method is that when the group of criteria is collapsed, the rank of this group can also be obtained, which can be used in classical decisionmaking methods. 
The rank of the group $\left(r_{x}\right)$ can be determined, for example, in the following ways:

1. $r_{x}=$ count of elements in a group $x$.

2. The sum of the correlation coefficients between the criteria that make up the group and the criterion $g$, which will replace the entire group:

$$
r_{x}=\sum_{i}^{i \in x} m_{i, g}
$$

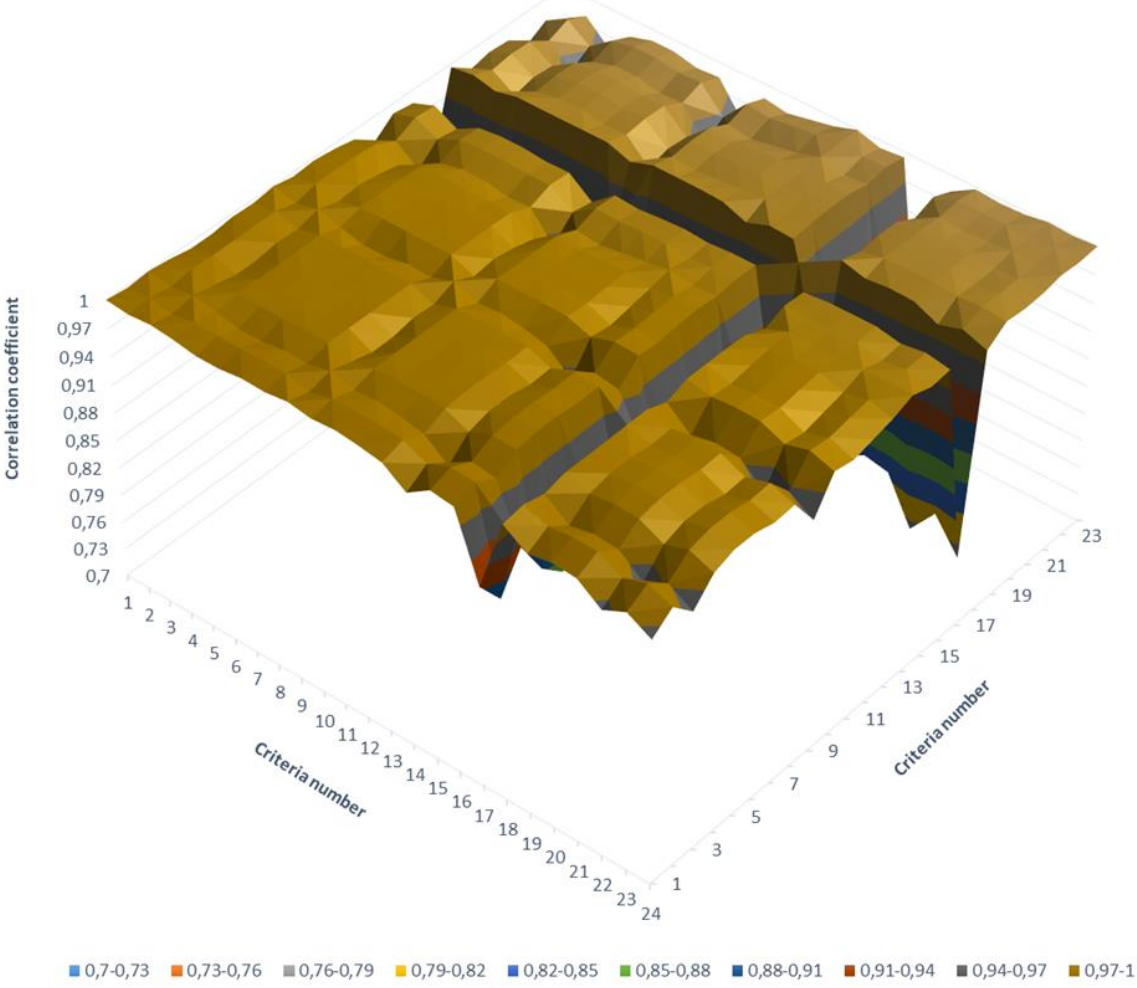

Fig. 3. Stereometric visualization of the surface of the criteria's correlation

Thus, as a result of applying this method, we get a new decision-making problem, characterized by the same choice set, but having a smaller number of criteria and supplemented by the ranks of these criteria: $A^{\prime}=\left\{a_{i}^{\prime}\right\}$, where $a_{i}^{\prime}=\left\{\left(a_{i, j} ; r_{j}\right)\right\}, 1 \leq j \leq K^{\prime} \leq K, K^{\prime}$ - new count of criteria.

\subsection{Visual Analytics of Stereometric Images of Multi-criteria Alternatives}

The effectiveness of research and analysis of stereometric images depends significantly on a foreshortening of view. Therefore, for this problem, it is important for the ability 
to change the foreshortening of the constructed stereometric image. At the same time, the analyst should have a convenient tool for making a quick change of foreshortening. The best way to do this is to construct a stereo model of the scene containing the corresponding visual images, and control the foreshortening with the camera, as is done in modern environments and 3D modeling tools.

Appropriate tools that ensure the implementation of the following functions should accompany visual analytics of stereometric images of multi-criteria alternatives:

- hide and show alternative;

- hide and show criterion;

- alternatives permutation;

- criteria permutation;

- selection of a specific alternative as a focused one (when using the method of visualizing alternatives as a single image);

- selection of a specific criterion as a focusable one (when using the method of visualizing criteria as a single images);

- in the case of visualizing alternatives with as a single image (Fig. 1), when focusing on a specific alternative, automatically change a color of remaining alternatives based on a level of similarity with the selected one (for example, by applying a gradient);

- in the case of visualization of criteria as a single image (Fig. 2) when choosing a criterion, automatically change a color of remaining criteria based on a level of similarity with the selected one;

- automatic reordering of alternatives depending on a level of similarity with the focusable alternative;

- automatic reordering of criteria depending on a level of similarity with the focusable criterion;

- in case of using the stereometric visualization method of criteria's correlation, provide an ability to customize a scales of a level of similarity for automatic grouping of criteria.

It is also possible to improve the quality of visual analytics of images obtained by the methods discussed above by using autostereoscopic displays [11].

\section{Experiment Description}

Consider the operation of the described methods for the problem of choosing the optimal solver OpenFoam [12,13]. Alternatively, consider three solvers - rhoCentralFoam, pisoCentralFoam, sonicFoam. The criteria are the results of calculating the deviation norm L1 of the numerical solution from the analytical one for the three-dimensional problem of inviscid flow around a cone. The computational problem was solved for following variants of initial parameters:

1. Mach number: $3,5,7$.

2. Cone half-opening angle (in degrees): 10, 15, 20. 
Methods for Construction and Research Stereometric Visual Images of Multi-criteria ... 7

3. Angle of attack (in degrees): 0, 5, 10.

In total, 24 values were calculated (for the combination of half-opening angle $=10^{\circ}$ and angle of attack $=10^{\circ}$, no calculations were performed).

To begin with, constructing a correlation surface and making criteria's permutation so that we clearly have groups (Fig. 4).

In this figure, the following groups (as the basic was using criterion with the maximum average correlation with other criteria of the group):

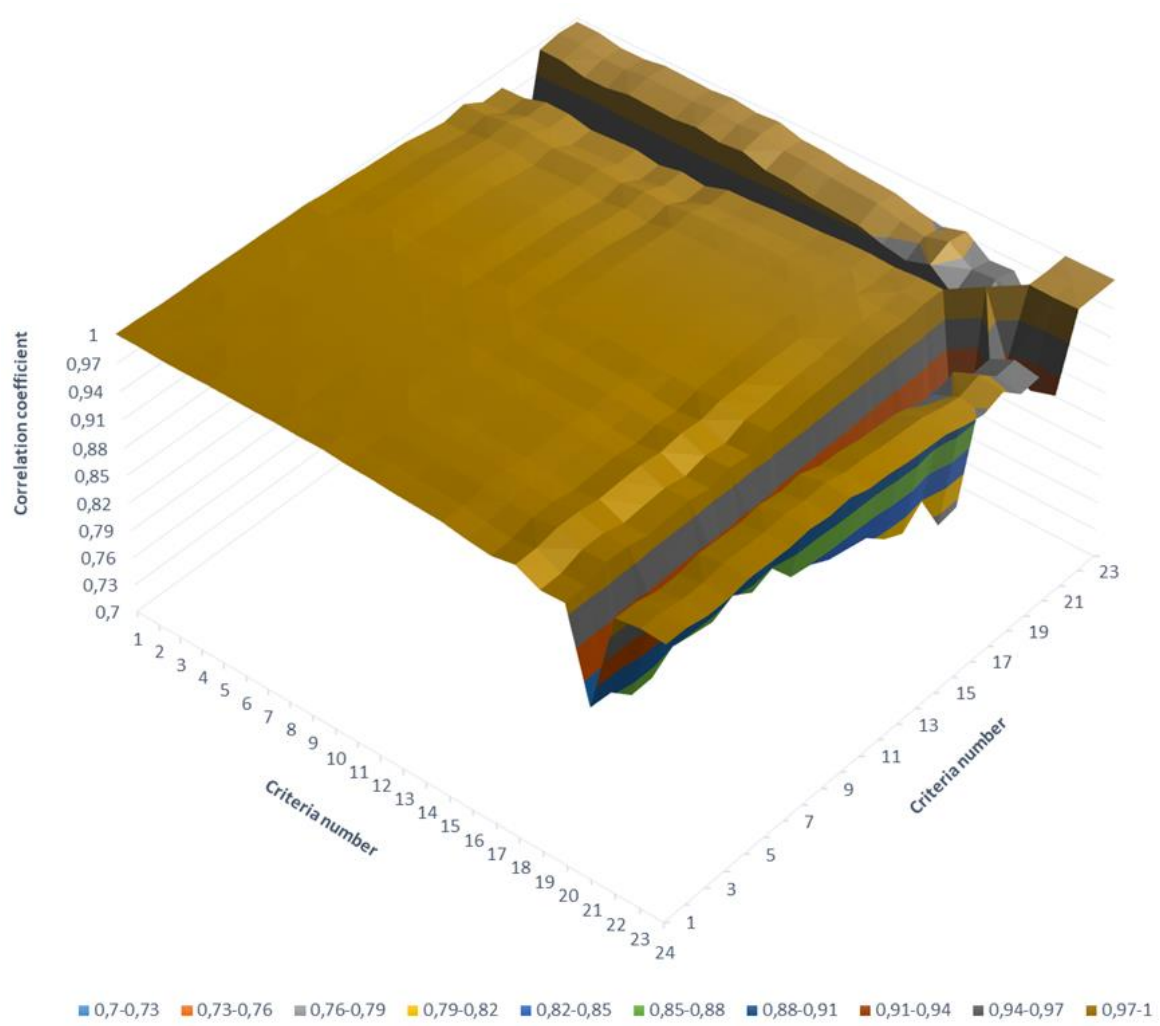

Fig. 4. The surface of the criteria's correlation

1. Criteria 1-12 (basic criterion -7 th).

2. Criteria 13-18 (basic criterion - 18th).

3. Criteria 19-20 (basic criterion -19 th).

4. Criterion 21.

5. Criteria 22-24 (basic criterion - 24th).

The ranks of the new criteria are as follows: $\{11.9966 ; 5.9983 ; 2 ; 1 ; 2.9995\}$. Visualize all three alternatives only for these remaining five criteria, sorted in descending order of rank (Fig. 5). As you can see in this figure, the far alternative (corresponds to the 
sonicFoam solver) is significantly worse than the other two (all columns below), i.e. it can be filtered.

\section{Discussion of Experimental Results}

The use of the developed methods for constructing and researching stereometric images of multi-criteria alternatives made it possible to reduce count of alternatives to two, and count of criteria to five. Thus, it was obtained the ranks of these criteria; one of the criteria (№ 21) has a substantially different correlation to the others, which may indicate that the corresponding computer experiment was performed incorrectly.

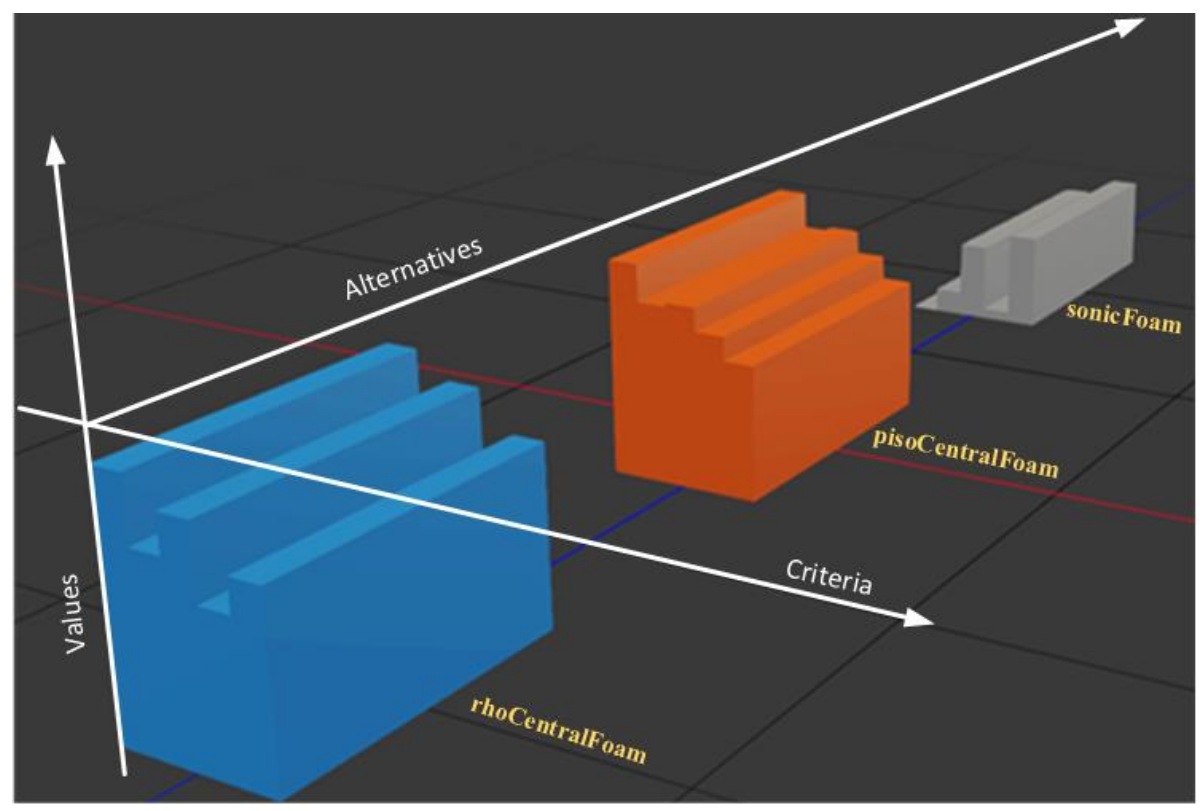

Fig. 5. Stereometric visualization of three alternatives after grouping criteria

Between the remaining two alternatives (Fig. 5) there is no longer such an unambiguous predominance, but traditional decision-making methods can already be applied to the choice of the best one. We solve this problem with four different decision-making methods - SMART, REGIME, PROMETHEE, TAXONOMY [2], and in all cases, we find that the solver rhoCentralFoam is the preferred alternative.

\section{Conclusions}

The developed methods for constructing and researching stereometric images of multicriteria alternatives allow solving the problems of reducing the set of alternatives, reducing the criteria by grouping them, as well as ranking the criteria. The use of the 
developed method for visualizing alternatives as single images allows for their visual filtering, and the use of the criteria visualization method as a single image in combination with the construction and analysis of the surface of the criteria's correlation allows grouping and ranking them. The experiment has demonstrated the effectiveness of the developed methods, since using visual analytics, we get a rationale for reducing the count of alternatives and criteria.

The development of methods for visualizing alternatives and criteria, as well as methods for their visual analytics and research, makes it possible to improve the quality and validity of the decisions obtained when choosing preferred alternatives and reduces the likelihood of errors when using traditional methods of decision-making by reducing the dimension of the original problem. It is possible to increase the efficiency of the described methods through the development and use of specialized software tools for visual analytics, which is one of the promising areas for the development of this topic.

\section{Acknowledgments}

The work was supported by Russian Science Foundation grant № 18-11-00215.

\section{References}

1. Castañón-Puga, M., Sanchez, M., Aguilar, L., Rodríguez-Díaz, A.: Applied Decision-Making. Applications in Computer Sciences and Engineering. Springer (2019). DOI: 10.1007/978-3-030-17985-4.

2. Alinezhad, A., Khalili, J.: New Methods and Applications in Multiple Attribute Decision Making (MADM). Springer, Cham (2019). DOI: 10.1007/978-3-030-15009-9.

3. Runkler T.: Data Visualization. In: Data Analytics. Springer Vieweg, Wiesbaden (2016). DOI: 10.1007/978-3-658-14075-5_4.

4. Podvesovskii. A.G., Isaev, R.A.: Visualization Metaphors for Fuzzy Cognitive Maps. Scientific Visualization 10(4), 13-29 (2018). - DOI: 10.26583/sv.10.4.02.

5. Zakharova, A.A., Shklyar, A.V.: Informative features of data visualization tasks. Scientific Visualization, 7 (2), 73-80 (2015).

6. Zakharova, A.A., Shklyar, A.V., Rizen, Y.S.: Measurable features of visualization task Scientific Visualization, 8 (1), 95-107 (2016).

7. Averchenkov, V.I., Miroshnikov, V.V., Podvesovskiy, A.G., Korostelyov, D.A.: Fuzzy and Hierarchical Models for Decision Support in Software Systems Implementations, A. Kravets et al. (Eds.). In: JCKBSE 2014, Communications in Computer and Information Science, vol. 466. pp. 410-421. Springer International Publishing (2014). DOI: 10.1007/978-3-31911854-3_35.

8. Figuera, J., Greco, S. Ehrgott, M. (Eds): Multiple Criteria Decision Analysis: State of the Art Surveys. Springer, New York (2005). - DOI: 10.1007/b100605.

9. Zakharova, A.A., Korostelyov, D.A., Fedonin, O.N.: Visualization Algorithms for Multicriteria Alternatives Filtering. Scientific Visualization 11(4), 66-80 (2019). DOI: 10.26583/sv.11.4.06.

10. Cohen, J., Cohen, P., West, S., Aiken, L.: Applied Multiple Regression/Correlation Analysis for the Behavioral Sciences. Routledge, New York (2003). DOI: 10.4324/9780203774441 
10 A. Zakharova, D. Korostelyov

11. Andreev, S.V., Bondarev, A.E., Bondareva, N.A. Stereo images of error surfaces in problems of numerical methods verification. Scientific Visualization. 12(2), 151-157 (2020). DOI: $10.26583 / \mathrm{sv} \cdot 12.2 .12$.

12. Bondarev, A.E., Kuvshinnikov, A.E. Analysis of the Accuracy of OpenFOAM Solvers for the Problem of Supersonic Flow Around a Cone. In: ICCS 2018, LNCS, vol. 10862. pp. 221-230. Springer, Cham (2018). DOI: 10.1007/978-3-319-93713-7_18.

13. Bondarev, A., Kuvshinnikov, A.: Comparative Estimation of QGDFoam Solver Accuracy for Inviscid Flow Around a Cone. In: IEEE The Proceedings of the 2018 Ivannikov ISPRAS Open Conference (ISPRAS-2018). pp. 82-87 (2018). DOI: 10.1109/ISPRAS.2018.00019. 